nephron

Practice
Nephron 2016;132:198-206

DOI: $10.1159 / 000444148$
Received: October 2, 2015

Accepted after revision: January 19, 2016 Published online: February 10, 2016

\title{
Tolerability and Healthcare Utilization in Maintenance Hemodialysis Patients Undergoing Treatment for Tuberculosis-Related Conditions
}

\author{
Abdurrahman M. Hamadaha Lynn M. Beaulieu ${ }^{a} \quad$ John W. Wilson ${ }^{b}$ \\ Timothy R. Aksamit ${ }^{c}$ James R. Gregoire ${ }^{a}$ Amy W. Williams ${ }^{a}$ John J. Dillon ${ }^{a}$ \\ Robert C. Albright ${ }^{a}$ Macaulay Onuigbo ${ }^{a}$ Venkateshwaran K. lyer ${ }^{a}$ \\ LaTonya J. Hickson a, d \\ ${ }^{a}$ Division of Nephrology and Hypertension, ${ }^{b}$ Infectious Diseases, and ${ }^{c}$ Pulmonary and Critical Care Medicine, \\ Department of Internal Medicine, ${ }^{d}$ Robert D. and Patricia E. Kern Center for the Science of Health Care Delivery, \\ Mayo Clinic, Rochester, Minn., USA
}

\section{Key Words}

Dialysis · End-stage renal disease · Healthcare utilization ·

Hospitalization · Maintenance hemodialysis

\begin{abstract}
Background: The incidence of tuberculosis (TB) in end-stage renal disease is significantly higher than that in the general population. Among those with kidney dysfunction, anti-TB treatment is associated with increased side effects, but the effect on healthcare utilization is unknown. Methods/Aim: To assess patient-reported symptoms, adverse effects and describe changes in healthcare utilization patterns during treatment for TB, we conducted a case series $(n=12)$ of patients receiving maintenance hemodialysis (HD) from Mayo Clinic Dialysis Services and concurrent drug therapy for TB from January 2002 through May 2014. Healthcare utilization (hospitalizations and emergency department (ED) visits independent of hospital admission) was compared before and during treatment. Results: Patients were treated for latent
\end{abstract}

$(n=7)$ or active $(n=5)$ TB. The majority of patients with latent disease were treated with isoniazid $(n=5,71 \%)$, while activedisease patients received a 4-drug regimen. Adverse effects were reported in $83 \%$ of patients. Compared to measurements prior to drug initiation, serum albumin and dialysis weights were similar at 3 months. Commonly reported antiTB drug toxicities were described. More than half (58\%) of the patients were hospitalized at least once. No ED or hospital admissions occurred in the period prior to drug therapy, but healthcare utilization increased during treatment in the latent disease group (hospitalization rate per person-month: pre 0 vs. post 1). Conclusions: Among HD patients, anti-TB therapy is associated with frequently reported symptoms and increased healthcare utilization. Among this subset, patients receiving treatment for latent disease may be those with greatest increase in healthcare use. Careful monitoring and early complication detection may help optimize medication adherence and minimize hospitalizations.

(c) 2016 S. Karger AG, Basel

\section{KARGER}

E-Mail karger@karger.com

www.karger.com/nef
(C) 2016 S. Karger AG, Basel

$1660-8151 / 16 / 1323-0198 \$ 39.50 / 0$
Dr. LaTonya J. Hickson

Division of Nephrology and Hypertension

Department of Medicine, Mayo Clinic, 200 First Street SW

Rochester, MN 55905 (USA)

E-Mail hickson.latonya@mayo.edu 


\section{Introduction}

According to the 2014 World Health Organization Global Report, tuberculosis (TB) remains a major public health concern. It is estimated that 9 million people developed TB in 2013 and 1.5 million died from the disease worldwide [1]. A particularly susceptible group of patients are those with end-stage renal disease (ESRD) receiving maintenance hemodialysis (HD) therapy [2]. The risk for dialysis patients developing TB can be up to 52 times higher than the general population depending on the geographic region [3-5]; the prevalence of TB may range from 1.6 to $5.8 \%$ in developed countries and upward of $28 \%$ in some developing countries [4]. The increased disease acquisition in ESRD is likely multifactorial but often attributed to decreased cellular immunity in the uremic state and a higher prevalence of high-risk comorbidities such as advanced age, diabetes and low body mass index [4, 6-10]. Importantly, survival is limited following $\mathrm{TB}$, with mortality rates ranging from 17 to $75 \%$ among dialysis-dependent patients $[4,11]$. In this patient group, clinical presentations of pulmonary TB disease may be atypical, given the high prevalence of extrapulmonary disease [12-14]. In addition, non-specific symptoms that may be masked by adverse effects from uremia and/ or chronic dialysis therapy may also conceal underlying TB disease $[3,10,15]$. Therefore, high mortality rates are often ascribed to delayed disease recognition leading to late diagnosis and subsequent treatment $[3,16]$. Additionally, ESRD increases risk of latent TB progression to active TB $[17,18]$.

Given the increased risk of TB acquisition among ESRD patients and prolonged time spent in healthcare settings, which may expose other high-risk persons, the Center for Disease Control and Prevention (CDC) has recommended that patients requiring maintenance dialysis should have at least one test for TB infection, with subsequent screening in the event of exposure, to aid in the detection of latent TB infection (LTBI) or subclinical disease [19]. However, there is no uniform screening approach for patients on dialysis [20]. Common amongst HD patients (20-70\%), LTBI is defined as a reactive tuberculin skin test or positive interferon gamma release assay in the absence of any clinical or radiographic evidence of active TB disease [16]. Treatment of LTBI requires isoniazid, rifampin or combination isoniazid plus rifapentine, administered over 3-9 months depending upon the specific $\operatorname{drug}(\mathrm{s})$ utilized [21]. Previously, 8 weeks of combination rifampin plus pyrazinamide was recommended as an alternative LTBI treatment option; however, due to higher rates of drug-associated hepatotoxicity, this combination regimen was no longer recommended after 2003 [22]. Unfortunately, anti-TB medications can produce many kinds of toxicities. This is particularly true in those with kidney failure, wherein the clearance of both parent drugs and their metabolites may be altered [12, 23, 24]. To address this, the American Thoracic Society published recommendations for various medication dosing adjustments in patients with renal dysfunction and those requiring dialysis [25]. Despite prevalent knowledge of the side effect profile of anti-TB treatment, treatment-related healthcare resource utilization such as emergency department (ED) visits and hospitalizations in this population has not been well-described. In this case series, we examined the major symptoms, adverse effect profile and frequency and healthcare resource utilization patterns in maintenance HD patients receiving anti-TB therapy at our outpatient HD facilities.

\section{Subjects and Methods}

\section{Study Overview}

The study cohort was identified from the Mayo Clinic institutional database. An initial query for patients $(n=2,952)$ with a diagnosis of any TB or mycobacterial infection was through international classification of disease (ICD) 9 codes (starting with 010, $011,012,013,014,015,016,017,018,031,137$ ) from January 1 , 2002, through May 31, 2014. Patients were then cross-referenced with ICD9 and billing codes for ESRD 585.6, dialysis V56.8, renal dialysis status or dependence on renal dialysis V45.11 or chronic kidney disease codes $(n=159)$. The final cohort $(n=12)$ was restricted to patients receiving maintenance HD therapy within one of Mayo Clinic Dialysis Services' 8 dialysis units from January 1, 2002 , to May 31, 2014, as previously described [26, 27]. Electronic chart reviews were conducted to confirm study eligibility and collect study data. Minnesota research authorization was available for all study participants. The Mayo Clinic Institutional Review Board approved the study.

\section{Patient Demographics and Covariables}

Patients' electronic medical records were reviewed for baseline demographics, kidney failure cause, laboratory values, anti-TB medications, changes to anti-TB therapy, documented adverse effects or new symptoms while on TB treatment as well as ED visits and hospitalizations.

\section{Definitions}

Active TB infection was defined as a positive culture of any tissue or specimen for Mycobacterium tuberculosis or positive acid-fast bacilli stain in the presence of typical symptoms and absence of atypical mycobacterial infection. A diagnosis of LTBI was based on a positive standard purified protein derivative (PPD) skin test or interferon gamma release (QuantiFERON; 
QFT $^{\circledR}$ ) test and no concomitant signs or symptoms of active infection or radiographic evidence of pulmonary TB. At our institution, the policy is to screen HD patients for LTBI at the initiation of dialysis with either PPD or QuantiFERON testing. Otherwise, only patients on the kidney transplant waiting list were screened annually during their transplant candidate evaluations. Active TB infection and LTBI treatment regimen selection did follow a standard protocol as outlined by the CDC $[22,25,28]$. Treatment for latent TB consisted of 6 or 9 months of single agent isoniazid, rifampin for 4 months or 8 weeks of combination rifampin plus pyrazinamide, which was an acceptable regimen prior to modified recommendations in 2003. Treatment of active TB was with 4-drug regimen of isoniazid, rifampin, ethambutol and pyrazinamide (initial phase), then isoniazid and rifampin for 4 months (continuation phase) for a total of 6 months. If necessary, the above regimens were modified at the discretion of the treating infectious disease specialist if toxicity or medical complications arose.

Symptoms experienced during anti-TB therapy were obtained through chart review of outpatient dialysis, specialty or primary care provider visits, dialysis nursing notes from each treatment and hospital or ED visits. Signs and symptoms assessed by providers or as reported by patients, particularly those leading to an ED visit or hospital admission, were recorded during the course of anti-TB therapy. Signs and symptoms, which were present prior to anti-TB therapy, were not recorded unless the intensity of the prior symptom was magnified during treatment course. Common toxicities of anti-TB therapy were recorded (when documented) including hepatitis (liver enzyme elevation), nausea, vomiting, peripheral neuropathy, peripheral blood cytopenias, skin rash and arthralgias. Other complaints were also recorded, such as lightheadedness or dizziness and syncope. Elevated liver enzymes were defined as values of alanine aminotransferase or aspartate aminotransferase above the upper limit of normal.

The primary outcome was healthcare utilization. Healthcare utilization was broadly defined as the need for hospital admission or ED care without hospitalization during the period of anti-TB therapy. Evaluations in the ED leading to hospitalization were counted as a hospital admission only. ED evaluations that resulted in dismissal from the ED were categorized as such. Hospitalization and ED utilization rates were calculated for each patient. To compare healthcare utilization patterns before and during treatment, hospitalization and ED visits were determined in the 6 months prior to treatment and during treatment. Overall, patient followup was restricted to the time the patient was both receiving anti-TB therapy and undergoing maintenance HD therapy at the Mayo Clinic Dialysis Services (MCDS). Therefore, patient follow-up ended at completion of anti-TB therapy, time of transfer to nonMCDS dialysis unit or discontinuation of dialysis (e.g. kidney transplantation).

\section{Statistical Analysis}

Data were expressed as mean and standard deviation for normally distributed data. Data that were not normally distributed were expressed as median and interquartile range. Event rates were defined as number of events per period at risk (per-personmonths). Statistical tests were not performed due to the small number of patients and frequency of multiple events in select patients.

\section{Results}

\section{Overall Cohort}

From January 2002 through May 2014, 12 patients receiving maintenance, in-center $\mathrm{HD}$ in MCDS were treated for LTBI $(n=7)$ or active TB $(n=5)$. Patient characteristics and drug therapy are provided in table 1 . The median age at treatment was 60 years (range 24-86), 75\% were men, $50 \%$ were white, mean dialysis vintage was 15.3 months, diabetes was the cause of ESRD in 50\%, mean weight at dialysis was $83 \mathrm{~kg}$ (range 45-110) and mean serum albumin was $3.2 \mathrm{~g} / \mathrm{dl}$ (range $2.1-4.2$ ). In the group of patients who were diagnosed with active $\mathrm{TB}, 60 \%$ had pulmonary TB and $40 \%$ had extrapulmonary TB. The majority (71\%) of LTBI patients were treated with isoniazid monotherapy. As per the published CDC guidelines, $100 \%$ of $\mathrm{HD}$ patients taking isoniazid also received pyridoxine [28]. Major symptoms, adverse effects and healthcare utilization patterns during anti-TB therapy are shown in table 2 . All active TB patients reported at least one adverse effect, whereas 5 of 7 (71\%) LTBI patients experienced adverse effects. The most frequent adverse effects overall were syncope (42\%), lightheadedness/dizziness (42\%), peripheral neuropathy (25\%) and liver enzyme elevation or abnormal liver function tests (LFTs; $17 \%)$. These adverse effects led to therapy modifications in only 3 patients $(25 \%)$. Over the initial course of treatment, serum albumin and weight changes were minimal with the exception of Patient 7, who lost $8 \mathrm{~kg}$ (table 3 ). Over half (58\%) of the patients were hospitalized at least once (median visits 1; range 1-3). However, ED evaluations without a hospital stay for complications or symptoms experienced during the treatment period were common (25\%; median visits 1 ; range $1-3$ ), particularly in the LTBI patient group. The overall number of hospitalizations, ED visits (only) and anti-TB medication changes during treatment are illustrated in figure 1 for LTBI and active TB patient groups.

\section{Major Symptoms and Adverse Effects Profile LTBI Group}

Among those treated for LTBI $(n=7)$, anti-TB treatment consisted of isoniazid monotherapy $(n=5)$ or was rifampin based $(n=2)$. During anti-TB therapy, the most frequent complaints were lightheadedness (43\%) and syncope $(43 \%)$, which often lead to additional healthcare use. One LTBI patient (Patient 2) discontinued treatment after 6 weeks due to elevated LFTs attributed to isoniazid toxicity. Only one LTBI patient reported no adverse symptoms and appeared to tolerate the treatment regimen. 
Table 1. Patient characteristics and anti-TB drug therapy in chronic HD patients with latent and active TB

\begin{tabular}{|c|c|c|c|c|c|c|c|c|c|c|}
\hline Patient & $\begin{array}{l}\text { Age, } \\
\text { years }\end{array}$ & Sex & $\begin{array}{l}\text { Dialysis } \\
\text { vintage, } \\
\text { months }\end{array}$ & $\begin{array}{l}\text { ESRD } \\
\text { cause }\end{array}$ & $\begin{array}{l}\text { Treatment } \\
\text { duration, } \\
\text { months }\end{array}$ & Isoniazid* & Pyrazinamide* & Rifampin* & Ethambutol* & $\begin{array}{l}\text { Treatment change } \\
\text { or discontinuation of } \\
\text { original prescription }\end{array}$ \\
\hline 1 & 52 & M & 26 & DM & 9 & $300 \mathrm{D}$ & - & - & - & No \\
\hline 2 & 59 & M & 1 & $\mathrm{DM}$ & $1.5^{¥}$ & $300 \mathrm{D}$ & - & - & - & Yes \\
\hline 3 & 63 & M & 45 & Unknown & 4 & $300 \mathrm{D}$ & - & - & - & No \\
\hline 5 & 86 & M & 7 & HTN & 9 & $300 \mathrm{D}$ & - & - & - & No \\
\hline 6 & 57 & M & $0^{\pi}$ & HTN & 2 & - & $2,0003 \mathrm{~W}$ & $600 \mathrm{D}$ & - & No \\
\hline 7 & 48 & M & 10 & $\mathrm{DM}$ & 4 & - & - & $600 \mathrm{D}$ & - & No \\
\hline \multicolumn{11}{|c|}{ Active TB } \\
\hline 8 & 24 & M & 3 & Unknown & 6 & $9003 W$ & $1,5002 \mathrm{~W}$ & $6002 \mathrm{~W}$ & $1,2003 \mathrm{~W}$ & No \\
\hline 9 & 61 & M & $-3^{\sim}$ & ATN & 6 & $600 \mathrm{D}$ & $2,000 \mathrm{D}$ & $900 \mathrm{D}$ & $1,600 \mathrm{D}$ & No \\
\hline
\end{tabular}

GN = Glomerulonephritis; HTN = hypertension; $\mathrm{DM}=$ diabetes mellitus; $\mathrm{D}=$ daily; $2 \mathrm{~W}=$ twice weekly; $3 \mathrm{~W}=$ thrice weekly.

"Dialysis started at same week of diagnosis of TB.

$\sim$ TB was diagnosed 3 months before starting dialysis but patient continued to receive treatment while on dialysis.

$¥$ The duration reflects the time patients were undergoing both chronic hemodialysis and treatment for TB. Shorter treatment duration indicates that patients received transplant or were no longer on dialysis.

Drug dosage is reported in milligrams. * If dose information not reported, the patient did not receive this medication as a part of the regimen.

Standard treatment regimen options for LTBI during the study period: isoniazid for 6 or 9 months, rifampin for 4 months, rifampin and pyrazinamide (combination no longer supported by the CDC beginning in 2003 due to reports of liver injury) for 2 months duration. http://www.cdc.gov/tb/topic/treatment/ltbi.htm.

Standard treatment regimen options for active TB during the study period included combination regimens of isoniazid, rifampin, pyrazinamide and ethambutol (initial phase) followed by isoniazid and rifampin (continuation phase). http://www.cdc.gov/tb/topic/ treatment/tbdisease.htm.

Dose adjustments and other alterations: pyrazinamide and ethambutol require dosing adjustments for renal insufficiency. Isoniazid is provided at standard dosing regimens of either $300 \mathrm{mg} /$ day or $900 \mathrm{mg}$ thrice weekly.

\section{Active TB Group}

Among those treated for active TB $(\mathrm{n}=5)$, anti-TB therapy comprised a 4-drug isoniazid-based regimen. The most frequently reported symptoms during anti-TB therapy were peripheral neuropathy $(40 \%)$, syncope (40\%) and light headedness (20\%). One patient in this group developed confusion. Two active TB patients (40\%) required a medication change or discontinuation of treatment. Patient 10 developed severe peripheral neuropathy and muscle weakness 6 weeks after starting treatment with rifampicin, isoniazid, ethambutol and pyrazinamide and opted to temporarily discontinue the treatment. $\mathrm{Pa}$ tient 12 developed significant peripheral neuropathy and elevated LFTs. His isoniazid and ethambutol were held more than once during the treatment course, and then reintroduced at a later date, with a 6-month total duration of therapy.

\section{Healthcare Utilization before and after Anti-TB Therapy}

Healthcare utilization defined as hospitalization or ED visit without hospital admission are illustrated in figure $2 a$ and $b$ by TB treatment group and timing of TB therapy. The LTBI patients had no ED visits or hospitalizations prior to anti-TB therapy, but these events increased after treatment was started. While most patients had new onset of symptoms during anti-TB therapy, 3 patients (2-LTBI and 1-active) had the highest healthcare utilization rates and accounted for the majority of hospital and ED visits, as illustrated in table 3.

\section{LTBI Group}

Among the LTBI patients, $43 \%$ were hospitalized (median visits 2 ; range $1-3$ ) and $26 \%$ were evaluated in the ED (median visits 2; range 1-3) for complications or 
Table 2. Symptomatology, adverse effects and healthcare utilization during anti-TB therapy

\begin{tabular}{|c|c|c|c|c|c|c|c|}
\hline Patient & Anti-TB drugs & $\begin{array}{l}\text { Major } \\
\text { symptoms or } \\
\text { adverse effects }\end{array}$ & $\begin{array}{l}\text { Other symptoms or } \\
\text { adverse effects }\end{array}$ & $\begin{array}{l}\text { Hospitalization, } \\
\mathrm{n}\end{array}$ & $\begin{array}{l}\text { Cause of } \\
\text { hospitalization }\end{array}$ & $\begin{array}{l}\mathrm{ED} \\
\text { visits, } \mathrm{n}\end{array}$ & $\begin{array}{l}\text { Cause of } \\
\text { ED visits }\end{array}$ \\
\hline 1 & INH & Seizure & Lightheadedness & Yes (3) & $\begin{array}{l}\text { Subdural hematoma, } \\
\text { seizures, hyperkalemia }\end{array}$ & No & - \\
\hline 3 & INH & $\begin{array}{l}\text { Peripheral } \\
\text { neuropathy }\end{array}$ & - & No & - & No & - \\
\hline 4 & INH & Syncope & $\begin{array}{l}\text { Muscle weakness } \\
\text { dizziness, elevated } \\
\text { LFTs, N/V }\end{array}$ & No & - & No & - \\
\hline 7 & Rifampin & - & - & No & - & No & - \\
\hline \multicolumn{8}{|c|}{ Active TB } \\
\hline 8 & $\begin{array}{l}\text { INH, rifampin, } \\
\text { PZA, ethambutol }\end{array}$ & Chest pain & $\mathrm{N} / \mathrm{V}$ & Yes (1) & Chest pain & No & - \\
\hline 9 & $\begin{array}{l}\text { INH, rifampin, } \\
\text { PZA, ethambutol }\end{array}$ & Syncope & - & Yes (1) & Syncope & No & - \\
\hline 10 & $\begin{array}{l}\text { INH, rifampin, } \\
\text { PZA, ethambutol }\end{array}$ & $\begin{array}{l}\text { Peripheral } \\
\text { neuropathy }\end{array}$ & $\begin{array}{l}\text { Muscle weakness } \\
\text { confusion }\end{array}$ & No & - & No & - \\
\hline 11 & $\begin{array}{l}\text { INH, rifampin, } \\
\text { PZA, ethambutol }\end{array}$ & Syncope & - & Yes (1) & Syncope & Yes (1) & Syncope \\
\hline
\end{tabular}

$\mathrm{INH}=$ Isoniazid; N/V = nausea and vomiting; PZA = pyrazinamide; $\mathrm{n}=$ number of hospitalizations or ED visits. ED visits indicate ED visits that did not lead to admission, which is otherwise reported under hospitalization.

symptoms experienced during the treatment period. In the preceding 6 months, LTBI patients had no hospitalizations or ED visits. However, after initiation of LTBI therapy, these events increased. Notably, Patient 2 received isoniazid monotherapy and was hospitalized twice and had 3 additional ED visits. His complaints were primarily gastroenterological, such as abdominal pain and constipation, but 2 hospitalizations were for syncope, lightheadedness and elevated LFTs. His treatment was aborted before completion. Despite the high healthcare utilization rate, only one patient had a medication change in the LTBI group. Another patient (Patient 1) at 14 weeks into treatment was hospitalized 64 days after developing a subdural hematoma complicated by recurrent seizures and physical debility. This patient accounted for $50 \%$ of the total LTBI group hospitalizations.

\section{Active TB Group}

Among the active TB patients, a majority (80\%) was admitted to the hospital at least once (median visits 1; range 1-3). Presenting symptoms and diagnoses upon hospital admissions included syncope, muscle weakness, pancreatitis and chest pain. In the active TB group, total numbers of hospital and ED visits were similar in the 6 months prior to and during treatment for TB. However, Patient 12 had the highest hospitalization rate $(0.02$ per- 
Table 3. Healthcare utilization patterns before and after anti-TB therapy initiation

\begin{tabular}{|c|c|c|c|c|c|c|c|c|c|c|c|c|c|}
\hline : & $\begin{array}{l}\stackrel{\infty}{\Xi} \\
\stackrel{\Delta}{\Delta} \\
\stackrel{\infty}{4}\end{array}$ & $\stackrel{凶}{\infty}$ & 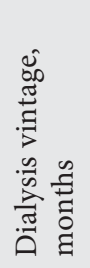 & 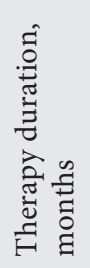 & 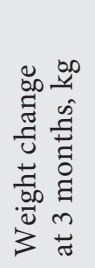 & 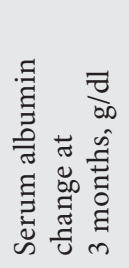 & 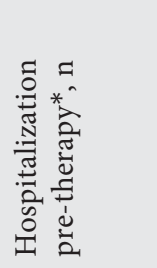 & 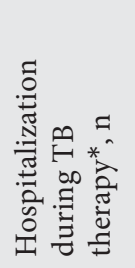 & 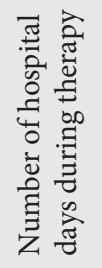 & 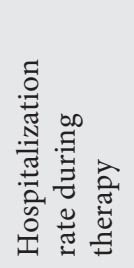 & 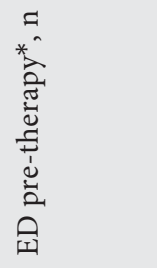 & 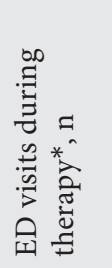 & 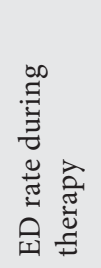 \\
\hline 1 & 52 & M & 26 & 9 & 0.7 & -1.3 & No & Yes (3) & 64 & 0.014 & No & No & 0 \\
\hline 2 & 59 & M & 1 & 1.5 & -0.6 & 1.2 & No & Yes (2) & 11 & 0.059 & No & Yes (3) & 0.07 \\
\hline 3 & 63 & M & 45 & 4 & 1.9 & $\mathrm{n} / \mathrm{a}$ & No & No & 0 & 0 & No & No & 0 \\
\hline 4 & 71 & $\mathrm{~F}$ & 47 & 9 & 1.0 & -0.1 & No & No & 0 & 0 & No & No & 0 \\
\hline 5 & 86 & M & 7 & 9 & 3.5 & -0.2 & No & No & 0 & 0 & No & No & 0 \\
\hline 6 & 57 & M & $0^{\pi}$ & 2 & 2.2 & $\mathrm{n} / \mathrm{a}$ & Unknown & Yes (1) & 4 & 0.018 & Unknown & Yes (1) & 0.017 \\
\hline \multicolumn{14}{|l|}{ Active $T B$} \\
\hline 9 & 61 & M & $-3^{\sim}$ & 6 & 10.6 & $\mathrm{n} / \mathrm{a}$ & Yes (1) & Yes (1) & 69 & 0.0090 & No & No & 0 \\
\hline 10 & 66 & $\mathrm{~F}$ & 8 & 1.5 & 0.5 & $\mathrm{n} / \mathrm{a}$ & No & No & 0 & 0 & No & No & 0 \\
\hline 11 & 84 & M & 7 & 6 & 0.7 & -0.6 & No & Yes (1) & 7 & 0.0058 & Yes (1) & Yes (1) & 0.0056 \\
\hline 12 & 43 & $\mathrm{~F}$ & 30 & 6 & 0 & 0.7 & Yes (1) & Yes (3) & 13 & 0.018 & No & No & 0 \\
\hline Total & - & - & - & 25.5 & 2.86 & 0.002 & 6 & 6 & 91 & 0.0089 & 1 & 1 & 0.0013 \\
\hline Entire cohort & - & - & - & 64.0 & 1.25 & 0.1 & 6 & 12 & 170 & 0.0069 & 1 & 5 & 0.0026 \\
\hline
\end{tabular}

ED visits are visits that did not lead to admission, which is otherwise reported under hospitalization; N/V = nausea and vomiting; $\mathrm{n}=$ number of hospitalizations or ED visits; $\mathrm{n} / \mathrm{a}=$ data not available. Rate is expressed as ED visits (hospitalization) - per-person-day (to change to per-person-month multiply these results * 30 ).

* In the 6 months prior to initiation of anti-TB treatment, for those who have been on dialysis for $<6$ months, only admissions/ED visits during the period on dialysis was included.

$\sim$ Patient was not followed at our institution.

" Dialysis started at same week of diagnosis of TB.

Fig. 1. Number of hospitalizations, ED visits (only) and anti-TB medication change among dialysis patients receiving treatment for latent $(n=7)$ or active $(n=5)$ TB.

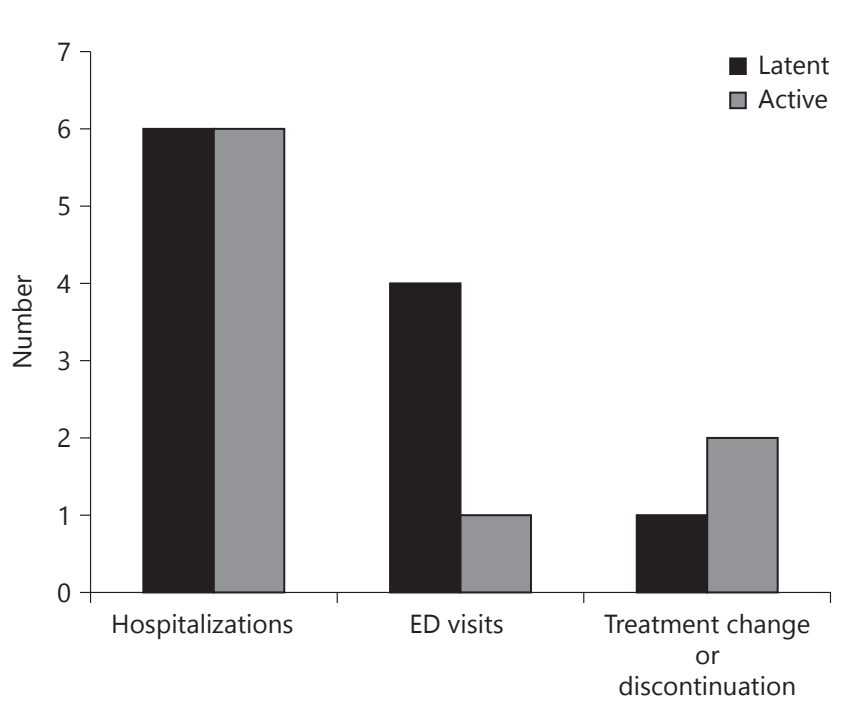




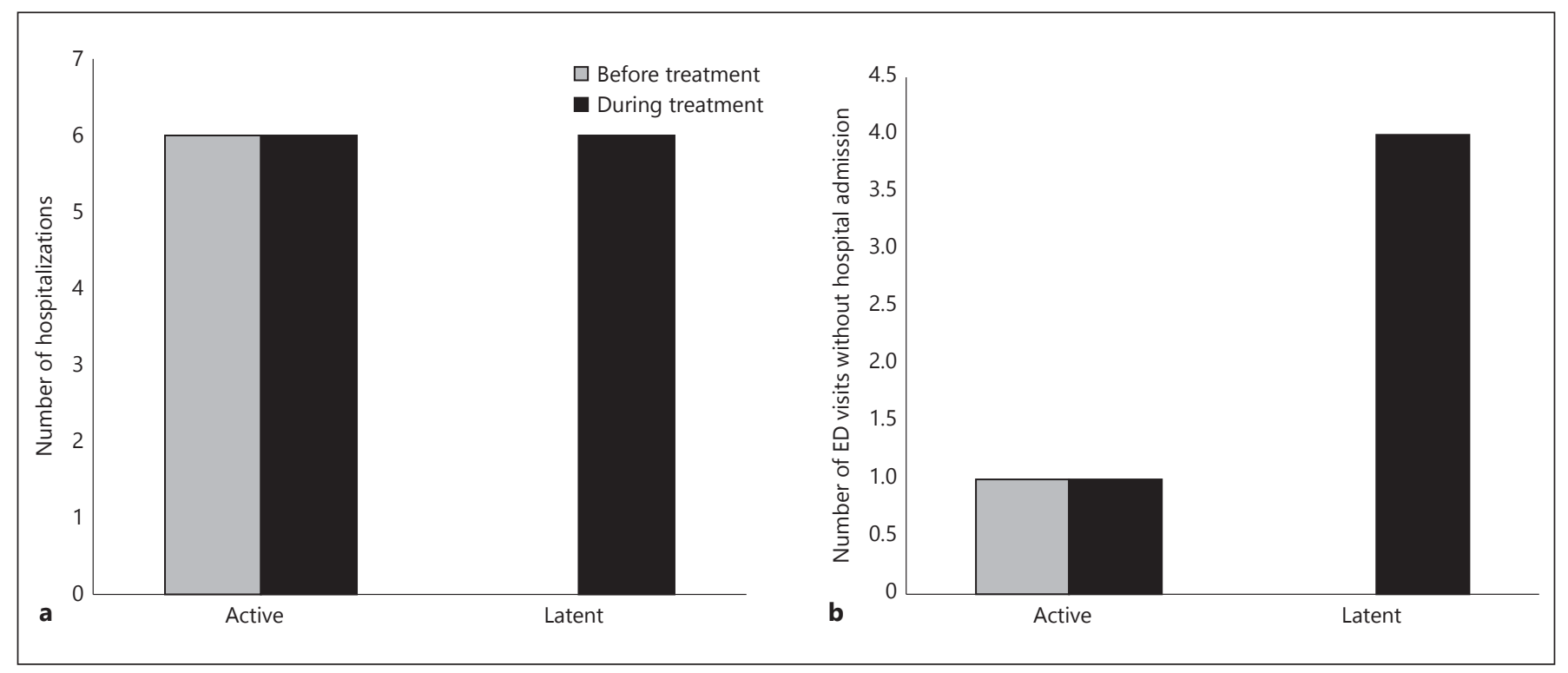

Fig. 2. a Number of hospitalizations 6 months prior to initiation and during anti-TB therapy by active $(n=5)$ and latent $(n=7)$ TB groupings. b Number of ED visits (without hospitalization) 6 months prior to initiation and during anti-TB therapy by active ( $\mathrm{n}=5$ ) and latent $(\mathrm{n}=7)$ TB groupings.

patient-day or 0.54 per-person-month) and was admitted to the hospital 3 times during the treatment period, accounting for $50 \%$ of the total active TB group hospitalizations. Muscle weaknesses, peripheral neuropathy, abdominal pain, light headedness and elevated LFTs were all reported. The patient was eventually diagnosed with pancreatitis later complicated by recurrent pancreatitis episodes with no clear etiology.

\section{Discussion}

Our study reveals for the first time a pattern of increased healthcare utilization relative to pretreatment period and poor anti-TB therapy tolerance among patients receiving maintenance $\mathrm{HD}$. In particular, patients treated for LTBI had the most significant change in number of hospitalizations and $\mathrm{ED}$ visits when compared to the 6-month period directly preceding anti-TB treatment. Overall, only 2 of 12 (17\%) were free of patient-reported symptomatology during therapy. New onset of syncope, lightheadedness and neuropsychiatric toxicities accounted for $50 \%$ of complaints leading to hospitalization, and abdominal pain accounted for $60 \%$ of ED visits during the treatment period. However, other serious events, including pancreatitis and subdural hematoma, occurred. These findings illustrate the potential impact of anti-TB treat- ment on acute care service needs due to the toxicities of anti-TB therapy and/or heightened sensitivity to symptoms typically tolerated during the course of maintenance HD therapy.

The captured major symptom and adverse effect profile of patients in our study is similar to previous reports. Quantrill et al. [12] reported a high incidence of adverse effects related to TB medications leading to changes in drug treatment in 16 dialysis patients (14 peritoneal dialysis; $2 \mathrm{HD}$ ) treated for active TB. In comparison, in our study, only $25 \%$ of subjects were clinically deemed to require a medication change or discontinuation, although those with frequent acute care services use may have benefited from changes in therapy. Quantrill et al. [12] and group also reported neuropsychiatric side effects in 2 patients in the form of confusion and seizure activity potentially related to isoniazid neurotoxicity $[12,23]$. In our study, syncope, lightheadedness and neuropsychiatric effects accounted for $50 \%$ of hospitalizations, and abdominal pain accounted for $60 \%$ of $\mathrm{ED}$ visits. Syncope and lightheadedness were common among our cohort, which may relate to decreased tolerability of the hemodynamic changes and volume removal during HD despite minimal changes found in body weight during the treatment period. Notably, 1 patient developed acute pancreatitis, a complication that has been described with isoniazid [29]. From a healthcare 
utilization perspective, over one-half (58\%) of our patients had at least 1 hospitalization. In comparison, in the 6 months prior to treatment, hospitalizations were non-existent for the LTBI group, illustrating the increased usage of acute services. However, 3 patients accounted for 8 hospitalizations and $3 \mathrm{ED}$ visits, illustrating that some patients were less tolerant of adverse effects, leading to an increased need for acute care services. Overall, these findings suggest that in maintenance HD patients drug-related toxicities are common and of significant impact.

Given the increased risk of side effects in the dialysis population, the American Thoracic Society, the Infectious Diseases Society of America and the CDC have jointly published guidelines for the appropriate dosing and dosing intervals in this population to improve tolerance and decrease incidence of side effects [25]. As a group, patients with LTBI appeared to experience fewer symptoms and adverse effects during the study period, likely due to monotherapy with isoniazid or rifampin. However, some LTBI patients appeared to have poor drug tolerance leading to increased healthcare utilization, suggesting an exaggerated sensitivity to toxicities of antiTB therapy beyond that of other HD patients.

Our case series has some limitations. The small number of patients has limited the ability to fully explore the spectrum of drug toxicities and healthcare utilization patterns associated with anti-TB treatment in the HD population. Patients receiving maintenance HD represent a complex, high-risk population for morbidity and mortality. Therefore, symptom assessment may be blurred by the multiple comorbidities and natural course of underlying chronic disease progression such as diabetes. Future studies with a control group of dialysis and non-dialysis patients may allow for comparison of the true magnitude of adverse drug reactions, and healthcare utilization differences attributed to advanced chronic kidney disease and dialysis dependence. Understanding this limitation, we compared hospital and ED visit numbers pre-anti-TB therapy to the number during treatment to highlight differences related to initiation of therapy. While larger studies of HD patient populations would be useful to calculate healthcare utilization rates, if these same studies apply billing claims data, they may help confirm trends but will lack the granularity of detail, which can be obtained by individual chart review.

Overall, our findings raise concerns not only regarding the negative impact on the health of the patients, but also their quality of life, particularly during times requiring increased healthcare use and the increased cost of care at- tributed to anti-TB treatment. In particular, this may be of concern to outpatient dialysis facilities with a higher incidence of LTBI. Given the recent implementation of the Center for Medicare and Medicaid Services (CMS) Quality Incentive Program, hospital readmissions are being included as quality measures in outpatient dialysis facilities $[30,31]$. Based on the increased frequency of healthcare utilization found in our study, facilities with high LTBI or active TB rates may have a disadvantage due the potential risk for increased hospitalization (and 30day readmission) rates in their facilities. LTBI or active TB could be an important patient comorbidity risk adjustor when comparing the hospitalization and readmission rates. In addition, those requiring anti-TB treatments may be denied admission to dialysis facilities because of their increased resource utilization. However, further studies are needed to assess the impact of anti-TB therapy on morbidity, mortality and healthcare utilization in this population.

In conclusion, anti-TB therapy is associated with frequent symptoms and adverse effects leading to increased healthcare resource utilization in maintenance HD patients. Increased awareness of potential complications and careful monitoring of patients are essential to optimize medication adherence, recognize symptoms of intolerance early and understand when adjustments may be needed to dialysis treatments or medication regimens given symptomatology. Collectively, these efforts may help minimize healthcare utilization for acute care needs due to adverse effects of anti-TB therapies.

\section{Grant Support}

This project was supported by the Robert D. and Patricia E. Kern Center for the Science of Health Care Delivery for L.J.H. This publication was also supported by grant number UL1 TR000135 from the National Center for Advancing Translational Sciences. Its contents are solely the responsibility of the authors and do not necessarily represent the official views of the NIH.

\section{Disclosure Statement}

The results presented in this paper have not been published previously in whole or part, except in abstract format at the 17th International Conference on Dialysis, Advances in Kidney Disease 2015; January 28-30, New Orleans, Louisiana.

\section{Statement of Ethics}

This study was approved by the institutional review board. 


\section{References}

1 World Health Organization: Global Tuberculosis Report, 2014-2015.

-2 Al-Efraij K, Mota L, Lunny C, Schachter M, Cook V, Johnston J: Risk of active tuberculosis in chronic kidney disease: a systematic review and meta-analysis. Int J Tuberc Lung Dis 2015;19:1493-1499.

-3 Segall L, Covic A: Diagnosis of tuberculosis in dialysis patients: current strategy. Clin J Am Soc Nephrol 2010;5:1114-1122.

4 Unsal A, Ahbap E, Basturk T, Koc Y, Sakaci T, Sinangil Arar A, Kayabasi H, Sevinc M: Tuberculosis in dialysis patients: a nine-year retrospective analysis. J Infect Dev Ctries 2013;7: 208-213.

5 Ahmed AT, Karter AJ: Tuberculosis in California dialysis patients. Int J Tuberc Lung Dis 2004;8:341-345.

6 6 Hussein MM, Mooij JM, Roujouleh H: Tuberculosis and chronic renal disease. Semin Dial 2003;16:38-44.

7 Fang HC, Lee PT, Chen CL, Wu MJ, Chou KJ, Chung HM: Tuberculosis in patients with end-stage renal disease. Int J Tuberc Lung Dis 2004;8:92-97.

8 Christopoulos AI, Diamantopoulos AA, Dimopoulos PA, Goumenos DS, Barbalias GA: Risk factors for tuberculosis in dialysis patients: a prospective multi-center clinical trial. BMC Nephrol 2009;10:36.

-9 Klote MM, Agodoa LY, Abbott KC: Risk factors for mycobacterium tuberculosis in US chronic dialysis patients. Nephrol Dial Transplant 2006;21:3287-3292.

10 Abdelrahman M, Sinha AK, Karkar A: Tuberculosis in end-stage renal disease patients on hemodialysis. Hemodial Int 2006;10:360-364.

-11 Shu CC, Wu VC, Yang FJ, Pan SC, Lai TS, Wang JY, Wang JT, Lee LN: Predictors and prevalence of latent tuberculosis infection in patients receiving long-term hemodialysis and peritoneal dialysis. PLoS One 2012; 7:e42592.

12 Quantrill SJ, Woodhead MA, Bell CE, Hardy CC, Hutchison AJ, Gokal R: Side-effects of antituberculosis drug treatment in patients with chronic renal failure. Eur Respir J 2002; 20:440-443.

13 Yang WF, Han F, Zhang XH, Zhang P, Chen $\mathrm{JH}$ : Extra-pulmonary tuberculosis infection in the dialysis patients with end stage renal diseases: case reports and literature review. J Zhejiang Univ Sci B 2013;14:76-82.

14 Kazancioglu R, Ozturk S, Gursu M, Avsar U, Aydin Z, Uzun S, Karadag S, Tatli E, Sar F: Tuberculosis in patients on hemodialysis in an endemic region. Hemodial Int 2010;14: 505-509.

15 Ko YC, Lee CT, Cheng YF, Hung KH, Kuo CY, Huang CC, Chen JB: Hypercalcaemia and haemophagocytic syndrome: rare concurrent presentations of disseminated tuberculosis in a dialysis patient. Int J Clin Pract 2004;58: 723-725.

16 Rao TM, Ram R, Swarnalatha G, Santhosh Pai BH, Ramesh V, Rao CS, Naidu GD, Dakshinamurty KV: Tuberculosis in haemodialysis patients: a single centre experience. Indian J Nephrol 2013;23:340-345.

17 Getahun H, Matteelli A, Chaisson RE, Raviglione M: Latent mycobacterium tuberculosis infection. N Engl J Med 2015;372:2127-2135.

18 Sia IG, Wieland ML: Current concepts in the management of tuberculosis. Mayo Clin Proc 2011;86:348-361.

19 Jensen PA, Lambert LA, Iademarco MF, Ridzon R: Guidelines for preventing the transmission of mycobacterium tuberculosis in health-care settings, 2005. MMWR Recomm Rep 2005;54:1-141.

20 Dobler CC, McDonald SP, Marks GB: Risk of tuberculosis in dialysis patients: a nationwide cohort study. PLoS One 2011;6:e29563.

21 Horsburgh CR Jr, Rubin EJ: Clinical practice. Latent tuberculosis infection in the United States. N Engl J Med 2011;364:1441-1448.

22 Centers for Disease Control and Prevention (CDC); American Thoracic Society: Update: adverse event data and revised American thoracic society/CDC recommendations against the use of rifampin and pyrazinamide for treatment of latent tuberculosis infection United States, 2003. MMWR Morb Mortal Wkly Rep 2003;52:735-739.

23 Siskind MS, Thienemann D, Kirlin L: Isoniazid-induced neurotoxicity in chronic dialysis patients: report of three cases and a review of the literature. Nephron 1993;64:303-306.

24 Abbas MT, Khan FY, Sulimon S, Baidaa A: Encephalopathy secondary to isoniazid in patients on hemodialysis. Indian J Nephrol 2013;23:54-56.

25 American Thoracic Society CDC, Infectious Diseases Society of America: Treatment of Tuberculosis. Morbidity and Mortality Weekly Report, 2003, vol 52, pp 1-77.

26 Hickson LJ, Chaudhary S, Williams AW, Dillon JJ, Norby SM, Gregoire JR, Albright RC Jr, McCarthy JT, Thorsteinsdottir B, Rule AD: Predictors of outpatient kidney function recovery among patients who initiate hemodialysis in the hospital. Am J Kidney Dis 2015; 65:592-602.

27 Schoonover KL, Hickson LJ, Norby SM, Hogan MC, Chaudhary S, Albright RC Jr, Dillon JJ, McCarthy JT, Williams AW: Risk factors for hospitalization among older, incident haemodialysis patients. Nephrology (Carlton) 2013;18:712-717.

28 Targeted tuberculin testing and treatment of latent tuberculosis infection. American thoracic society. MMWR Recomm Rep 2000;49: $1-51$.

29 Rabassa AA, Trey G, Shukla U, Samo T, Anand BS: Isoniazid-induced acute pancreatitis. Ann Intern Med 1994;121:433-434.

30 Centers for Medicare and Medicaid Services (CMS): National Dry Run Report: Standardized Readmission Ratio (SRR) for Dialysis Facilities. ESRD Quality Measure Development, Maintenance, and Support, UMKECC, 2014.

31 Centers for Medicare and Medicaid Services (CMS): Readmissions Reduction Program, 2015. 